\title{
Sedentary behaviour and bone health in children, adolescents and young adults: a systematic review-supplementary presentation
}

\author{
J. B. Koedijk ${ }^{1}$ - J. van Rijswijk ${ }^{1}$ - W. A. Oranje ${ }^{2}$ J. P. van den Bergh ${ }^{1,3}$. \\ S. P. Bours ${ }^{4}$ H. H. Savelberg ${ }^{5}$ N. C. Schaper ${ }^{1}$
}

Received: 19 July 2017 / Accepted: 9 August 2017 /Published online: 6 September 2017

(C) The Author(s) 2017. This article is an open access publication

\begin{abstract}
Background: Sedentary behaviour (SB) is a potential risk factor for suboptimal bone deposition in youth. Results: Total SB was negatively associated with lower extremity bone outcomes, while no association was observed with total body bone outcomes. Insufficient evidence was found for an association between total SB and lumbar spine bone outcomes. Conclusion: This review highlights the heterogeneity of the available evidence and emphasizes the need for well-designed studies.
\end{abstract}

Keywords Adolescent · Bone · Child · Osteoporosis . Sedentary behaviour $\cdot$ Unloading

The electronic supplementary material presented here complements the article "Sedentary behaviour and bone health in children, adolescents and young adults: a systematic review" [1].

Electronic supplementary material The online version of this article (https://doi.org/10.1007/s00198-017-4195-9) contains supplementary material, which is available to authorized users.

J. B. Koedijk

j.koedijk@student.maastrichtuniversity.nl

1 Division of Endocrinology, Department of Internal Medicine, Maastricht University Medical Centre, PO Box 5800, 6202, AZ Maastricht, The Netherlands

2 Division of Endocrinology, Department of Internal Medicine, Elisabeth-TweeSteden Hospital, Tilburg, The Netherlands

3 Division of Endocrinology, Department of Internal Medicine, VieCuri Medical Centre, Venlo, The Netherlands

4 Division of Rheumatology, Department of Internal Medicine, Maastricht University Medical Centre, Maastricht, The Netherlands

5 Department of Human Biology and Human Movement Sciences, Maastricht University Medical Centre, Maastricht, The Netherlands

\section{Aim}

The aim of this systematic review was to examine the association between SB and bone health in children, adolescents and young adults [1].

\section{Key findings}

Based on studies that used DXA and quantitative ultrasound, we found moderate evidence for a negative association between objectively measured SB and lower extremity bone outcomes in schoolchildren that was independent of moderate-to-vigorous physical activity (MVPA). We calculated that $1 \mathrm{~h}$ less sedentary time per day is associated with the same effect on femoral neck bone mineral density (BMD) as 18 min of MVPA (based on one high-quality study). Based on high-quality studies that used DXA, there was strong evidence to suggest no association between objectively measured SB and total body bone outcomes in schoolchildren, and insufficient evidence for an association with lumbar spine bone outcomes. For questionnaires, none of these relationships were observed.

\section{Importance, timeliness and interest}

This systematic review suggests that objectively measured total sedentary time is negatively associated with bone outcomes of the lower extremities in schoolchildren. 
Although the magnitude of the effect size seems small, it may theoretically be relevant for bone accrual in the young, in particular when the SB is maintained over several years. Furthermore, this review highlights the heterogeneity of the available evidence (different bone-imaging tools, measurement of SB, anatomical sites assessed, outcome parameters, adjustment of results) and emphasizes the need for well-designed studies.

\section{Significance of findings}

Optimizing bone accrual in the young is important to minimalize the risk of osteoporosis in later life. If the effect of SB on lower extremity bone outcomes is confirmed in other studies, reducing sedentary time could be helpful to optimize bone accrual in children who are unable or less interested in PA.

\section{Compliance with ethical standards}

Conflicts of interest Joost Koedijk, Johannes van Rijswijk, Wilma Oranje, Joop van den Bergh, Sandrine Bours, HansSavelberg and Nicolaas Schaper declare that they have no conflict of interest.

Open Access This article is distributed under the terms of the Creative Commons Attribution-NonCommercial 4.0 International License (http:// creativecommons.org/licenses/by-nc/4.0/), which permits any noncommercial use, distribution, and reproduction in any medium, provided you give appropriate credit to the original author(s) and the source, provide a link to the Creative Commons license, and indicate if changes were made.

\section{References}

1. Koedijk JB, van Rijswijk J, Oranje WA, van den Bergh JP, Bours SP, Savelberg HH, Schaper NC (2017) Sedentary behaviour and bone health in children, adolescents and young adults: a systematic review. Osteoporos Int https://doi.org/10.1007/s00198-017-4076-2 